\title{
OPHTHALMIC CONSEQUENCES OF MID-FACIAL TRAUMA
}

\author{
G. N. DUTTON, ${ }^{1}$ I. AL-QURAINY, ${ }^{2}$ L. F. A. STASSEN ${ }_{3}^{3}$ D. M. TITTERINGTON, ${ }^{4}$ K. F. MOOS ${ }^{5}$ \\ A. EL-ATTAR, \\ Glasgow, Plymouth and Sunderland
}

\begin{abstract}
SUMMARY
Fractures of the mid-face are commonly accompanied by injury to the visual system. Three hundred and sixty three patients who had sustained mid-facial fractures were assessed prospectively for evidence of such injury. The data have been used to devise a scoring system for the maxillofacial surgeon in order to help identify those patients at risk of eye injury. The scoring system has been evaluated on a further cohort of 100 patients with a similar spectrum of injury and the sensitivity and specificity of the system have been determined. The results of these studies have been reported in the maxillofacial literature. This paper reviews the data and results obtained. In summary, impaired visual acuity with a comminuted or out blow fracture, a motility abnormality, or facial fracture combined with head injury, sufficient to cause both retrograde and post-traumatic amnesia, emerged as major risk factors which are indicative of an adverse ophthalmic outcome deemed to warrant referral. The scoring system which was developed from this data was found to have a sensitivity of $94.4 \%$ and a specificity of $89 \%$ for the detection of patients thought to merit ophthalmic assessment. Failure to assess central visual function as objectively as practicable in patients who have sustained mid-facial fractures may lead to potentially treatable ophthalmic pathology not being identified.
\end{abstract}

Mid facial trauma sufficient to give rise to a facial bone fracture may also injure the visual system. Estimates of the incidence of ocular disorders following midfacial fracture vary considerably and range between $1 \%{ }^{1}$ and $57 \% .^{2}$ These estimates depend upon referral practice, whether the studies were prospective ${ }^{3,4}$ or retrospective $e^{2,5,6}$ and the

'Tennent Institute of Ophthalmology, Western Infirmary, Glasgow G11 6NT.

${ }^{2}$ Royal Eye Infirmary, Apsley Road, Plymouth PL4 6PL.

${ }^{3}$ Department of Oral and Maxillofacial Surgery, Sunderland District General Hospital, Kayll Road, Sunderland, Tyne \& Wear SR4 7TP.

${ }^{4}$ Department of Statistics, University of Glasgow, Glasgow G12 8QQ.

${ }^{5}$ Department of Oral and Maxillofacial Surgery, Canniesburn Hospital, Bearsden, Glasgow G61 1QL.

Correspondence to: Dr. G. N. Dutton, FRCS, FCOphth, Tennent Institute of Ophthalmology, Western Infirmary, Glasgow G11 6NT. severity of ophthalmic pathology deemed to merit reporting. Reports written by maxillofacial and plastic surgeons give lower estimates than those written by ophthalmologists.

When eye injuries are severe, they can be easily identified by any clinician, but a number of injuries (such as angle recession and retinal tears) may potentially threaten visual function and are difficult to detect by the nonophthalmologist. It is important to be able to identify such patients so that correct referral can be made for appropriate treatment and with a view to thorough documentation for potential medicolegal claims.

We have carried out a detailed prospective ophthalmic assessment on a cohort of 363 patients sustaining midfacial fractures in order to determine the spectrum of ophthalmic pathology which may arise and to identify those individuals who would benefit from an ophthalmic opinion. ${ }^{7,8}$ These data have been used to devise a scoring system whereby the maxillofacial surgeon can use his observations (without recourse to detailed eye examination) to identify those patients who may benefit from an ophthalmic opinion. ${ }^{9}$ The system has subsequently been evaluated on a separate cohort of 100 similar patients. ${ }^{10}$

\section{PATIENTS AND METHODS}

\section{Patients}

Three hundred and sixty three patients who had sustained mid-facial or frontal bone fractures and who were treated at the Maxillofacial Unit, Canniesburn Hospital, Glasgow between April 1, 1985 and March 31, 1987 were admitted to the initial study. All patients with persistent pathology were followed up for at least one year. Patients with craniofacial fractures managed by the same team in the regional neurosurgical unit were only included if they were transferred to or followed up in Canniesburn Hospital.

Patients with major trauma were evaluated ophthalmologically within 48 hours of injury, whilst those with less severe injuries were assessed at the ensuing weekly ophthalmic clinic. At a later stage (between December 1989 and August 1990), 100 additional patients with identical entry criteria were evaluated in the same manner. 


\section{Clinical Assessment}

All patients underwent prospective assessment of their maxillofacial status and were subsequently assessed in detail by both an ophthalmologist and an orthoptist. All data concerning the facial injuries, the ophthalmic complications and the management were comprehensively documented and coded for subsequent statistical analysis.

\section{Statistical Analysis}

All data were coded and tabulated and most statistical analyses were carried out using the statistical package Minitab.

\section{Potential Predictors}

Seven sets of data were chosen as potential predictors for ophthalmic injury. These were selected on the basis that they may all be routinely recorded by the maxillofacial surgeon and may have a bearing on the ultimate ophthalmic outcome. They comprised:

1. Basic patient data: sex, age and month of injury

2. Cause of injury

3. Type of bone and associated soft tissue injury

4. Method(s) of surgical treatment

5. Ophthalmic data: Affected eye. Visual acuity

6. Ocular motility data: Symptoms of double vision or obvious signs of eye movement disorder

7. Associated conditions: Retrograde and post-traumatic amnesia and cranial nerve palsies

\section{Outcome Categories}

The types of potential ophthalmic injury were classified into three outcome categories. ' 'Non-referral cases' comprised such self-limiting disorders as bruising and swelling. 'Routine referral cases' included those patients with traumatic pupillary changes, traumatic cataract and impaired accommodation. 'Early referral cases' were those for whom urgent therapeutic intervention might be

\section{Types of Fracture}

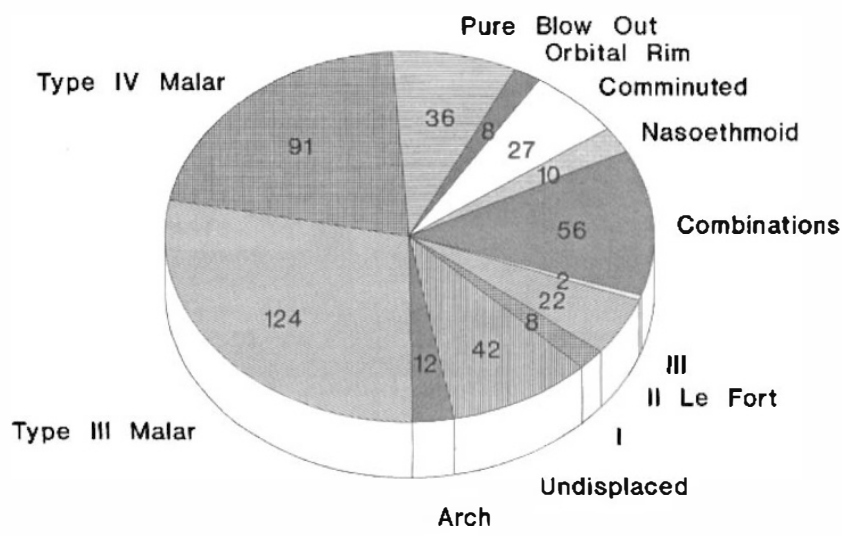

Fig. 1. Pie diagram illustrating the range of fracture types seen in the population studied. (Type IV malar fractures are characterised by frontozygomatic distraction whilst Type III malar fractures are not.) required, for example, retinal detachment and retro-bulbar haemorrhage. ${ }^{9}$

\section{Selection of Risk Factors}

Each of the potential predictors was initially considered separately with respect to each of the outcome categories. For each of the two-way contingency tables obtained, the Chi squared statistic was computed and those predictors which gave results at or near the $5 \%$ confidence level were noted.

\section{Construction of a Scoring System}

The data obtained above were analysed by means of stepwise linear regression analysis. This technique results in a selection of the subset of predictors which extracts the meaningful predictive information contained by all the predictors. The resulting subset therefore provides a parsimonious but efficient means of prediction. The coefficients of the corresponding regression equation were employed to derive the scores suitable for everyday practice.

\section{RESULTS}

\section{Details of Injuries Sustained}

Figure 1 illustrates the spectrum of mid-facial fractures sustained in the population studied and Figure 2 indicates the causes of the injuries sustained. The severity of eye injuries is illustrated in Figure 3. This was classified in a manner similar to the outcome criteria described by Holt and $\mathrm{Holt}^{2}$ in their extensive review of eye injuries sustained following facial fractures. Only 34 patients had no evidence of eye injury. Mild (reversible) disorders including eyelid swelling and subconjunctival haemorrhage was seen in 230 patients $(60 \%)$. Fifty seven patients had sustained eye injuries sufficient to cause visual dysfunction for a number of weeks including four cases of choroidal tears not involving the macula and 13 patients with macular oedema. The types of severe ophthalmic injury seen in 42 patients were as follows:

\section{CAUSES OF INJURY}

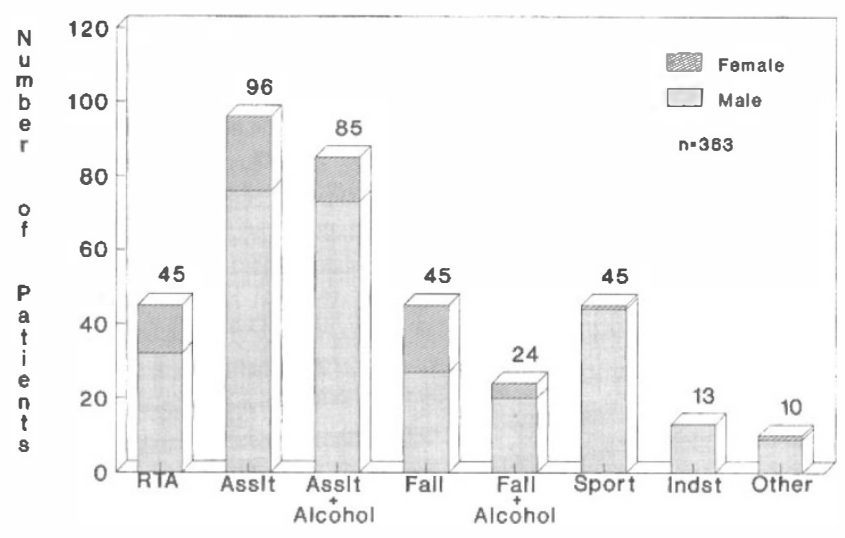

Fig. 2. Histogram showing the types of injuries sustained in the population studied. $(R T A=$ Road traffic accident, Asslt $=$ Assault. Indst = Industrial accident). Patients injured whilst under the influence of alcohol are illustrated separately. 
Retrobulbar haemorrhage

(requiring surgical intervention)

Corneal laceration

Hyphaema

Angle recession

Vitreous haemorrhage and/or retinal tears

Irreversible maculopathy

Optic nerve injury

\section{EYE INJURIES}

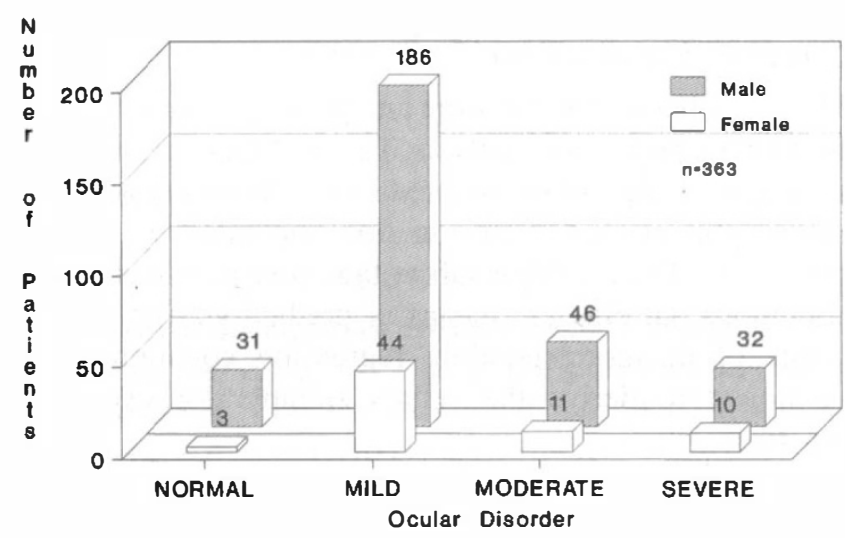

Fig. 3. Histogram showing the severity of the injuries sustained.

Although there were far fewer injured women than men, the proportion of women sustaining moderate or severe injuries was higher but not significantly so.

Figure 4 shows that 72 patients $(19.8 \%)$ were subject to diplopia as a sequel to their injury. Double vision was most common following road traffic accidents $(31 \%)$ and least common as a result of simple falls (10\%). Blow out fractures of the orbit led to diplopia in $58 \%$ of cases. Eighty two per cent of patients recovered from their double vision within six months of the injury. Only one patient (who had sustained a medial wall blow out fracture) ultimately required squint surgery for double vision.

\section{MAXILLOFACIAL INJURIES DIPLOPIA}

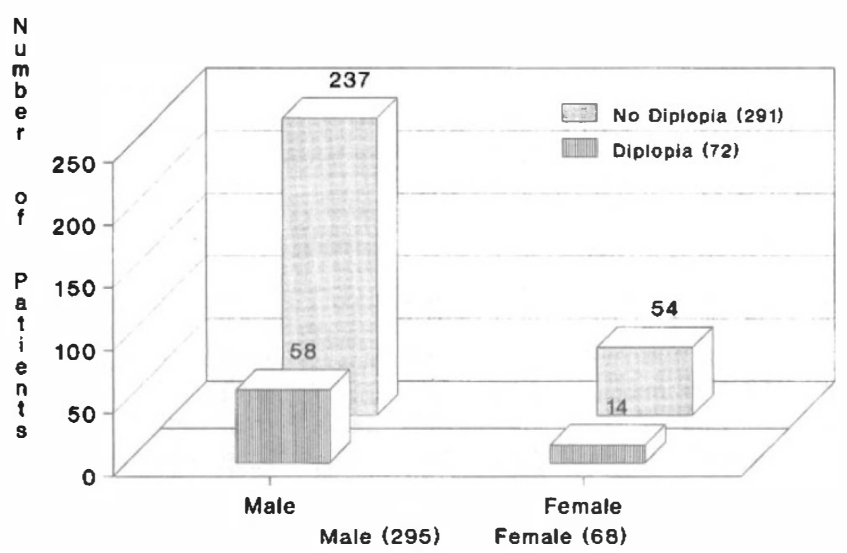

Fig. 4. Diagram illustrating the proportions of injured male and female patients experiencing diplopia at the time of presentation.

\section{Development of the Scoring System}

Not surprisingly, impairment in visual acuity emerged as the most sensitive, single predictor of eye injury.

Comminuted fractures of the malar culminated in the most severe eye and motility defects, no doubt as a sequel to the loss of the protection normally afforded by the lateral orbital wall.

Pure blow out fractures were commonly accompanied by motility defects warranting ophthalmic referral. Abnormalities of eye movement can, in most cases, easily be diagnosed by the maxillofacial surgeon primarily on the basis of diplopia. Although this predictor 'overlaps' with comminuted and blow out fractures, it emerged as a predictor which identified additional cases deemed to warrant referral.

Head injuries of sufficient severity to result in midfacial fractures accompanied by both retrograde and post traumatic amnesia also affect the visual system in many cases. This severe form of amnesia thus emerged as a significant risk factor. Figure 5 illustrates these risk factors, the scores apportioned, and their significance. The means whereby these values have been derived have been described previously. ${ }^{9}$

The tests for validity for these scores (when applied to the population from which they were derived) were found to be:

$\begin{array}{ll}\text { Sensitivity value } & =89 \% \\ \text { Specificity value } & =90 \%\end{array}$

Predictive value of a positive test $=92 \%$

Predictive value of a negative test $=87 \%$

The major cause for concern in this first series was six 'false negative' patients with peripheral vitreo-retinal pathology and six with angle recession who were only identified by detailed ophthalmic examination but did not have any other pathology sufficient to result in a high score.

\section{Evaluation of the Scoring System}

A further 100 patients with mid-facial fractures were MAXILLOFACIAL TRAUMA SCORE

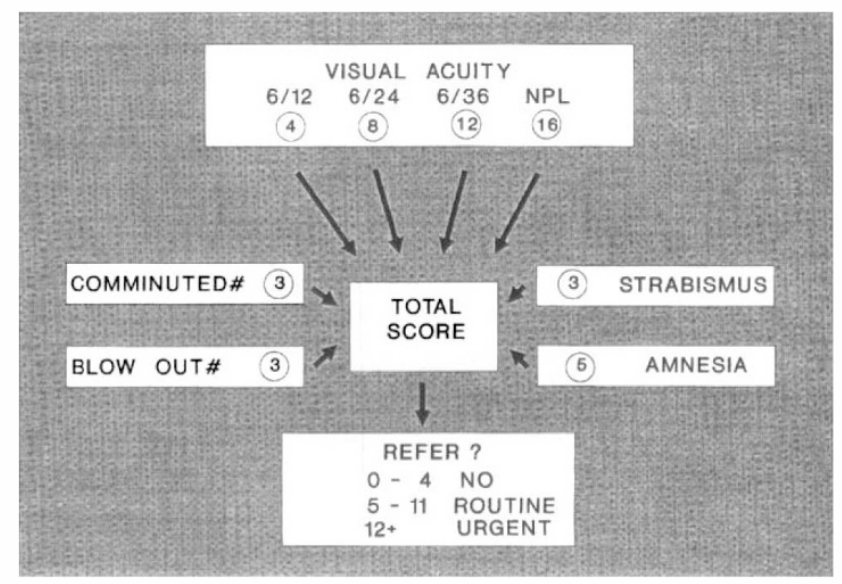

Fig. 5. Diagram showing the details of the scoring system. The circular numbers are the scores apportioned if the criterion is present. The term amnesia denotes both.retorgrade and post traumatic amnesia. 
tested using the above scoring system and also underwent prospective ophthalmic assessment. ${ }^{7}$ The above tests of validity were then carried out upon this new cohort.

Seventeen patients were correctly identified by the system as meriting ophthalmic referral (true positive) while 73 were found to be true negatives. Nine patients were incorrectly identified as requiring referral (false positives) and there was only one false negative identification of a patient with marked enophthalmos who had diplopia in the extremes in gaze giving him a score of three. Six of the false positive referrals were found to have been due to poor vision due to amblyopia and not the injury per se (the instructions on the scoring sheet have now been amended appropriately).

The tests for validity of the scores obtained are as follows: ${ }^{10}$

$\begin{array}{ll}\text { Sensitivity value } & =94.4 \% \\ \text { Specificity value } & =89 \%\end{array}$

Predictive value of a positive test $=65.4 \%$

Predictive value of a negative test $=98.6 \%$

\section{DISCUSSION}

A high proportion of patients sustaining mid-facial fracture sustained injury to the visual system. The results which we have obtained are comparable with those of Holt and Holt who studied the nature of eye injuries in a similar cohort of patients. ${ }^{2}$ Casualty officers, and maxillofacial and plastic surgeons who commonly deal with such trauma may have had little training in ophthalmology which may lead to potentially sight threatening ophthalmic pathology not being identified. The detection of disorders such as retinal detachment and glaucoma may lead to appropriate sight saving treatment whilst the detailed assessment and documentation of all pathology, whether it is treatable or not, is essential for the purposes of subsequent insurance or legal claims. The scoring system described has a sensitivity and a specificity in the region of $90 \%$.

In many centres, it may be practicable to refer all patients with mid-facial fracture for an ophthalmic opinion, but there are many units worldwide where inadequate staffing levels preclude this ideal option.

No matter what referral criteria are employed, there will always be needless referrals (false positives) and some patients with significant ophthalmic pathology may not be identified (false negatives). The selection of 'predictors' and 'outcomes' was inevitably empirical and is open to criticism. However, these were discussed with a number of maxillofacial and ophthalmic surgeons before being chosen and hopefully represent a consensus view from both specialties. It should be stressed that the scoring system which has been derived from our results is meant only to provide guidance as to how best patients should be managed and should not be considered as a substitute for clinical acumen and common sense!

It may be thought that other predictors can be identified which will effectively select additional patients for referral. When considered in isolation, such predictors do have a fair predictive value, for example, patients between the ages of 30-39 years were more likely to have sustained eye injuries than any other group, whilst patients who had been involved in road traffic accidents are similarly at greater risk. However, when these predictors are considered within the above scoring system they fail to identify any additional individuals warranting referral. This is appropriate because the predictors are closely interrelated. For example, comminuted fractures and combined facial and head injury complicated by post-traumatic with retrograde amnesia (which emerged as significant risk factors for eye injury) are both common sequels to road traffic accidents, and thus knowledge of the cause of injury fails to add anything to the ultimate 'score'.

To our knowledge, this is the first set of studies to be published in the maxillofacial literature ${ }^{7,9,10}$ which definitively establishes that failure to assess visual acuity will lead to eye injuries going undetected.

In many cases, eyelid swelling at the time of presentation may preclude accurate assessment of visual acuity and subsequent determination of central visual function when the swelling subsides may be omitted. Whether or not the scoring system described in this paper is ultimately adopted by clinicians dealing with facial fractures, the data presented are sufficiently persuasive to argue that the discipline of recording at least an estimate of central visual function in all patients who sustain mid-facial fractures is mandatory if underlying eye injuries are to be detected and treated appropriately.

\section{REFERENCES}

1. McCoy FJ, Chandler RA, Magnan CG Jr, Moor JR, Siemsen G: An analysis of facial fractures and their complications. Plast Reconstruct Surg 1962, 29: 381-91.

2. Holt JE, Holt GR, Blodgett JM: Ocular injuries sustained during blunt facial trauma. Ophthalmology 1983, 90: $14-18$.

3. Miller GR and Tenzel RR. Ocular complications of midfacial fractures. Plast Reconstruct Surg 1967, 39: 37-42.

4. Jabaley ME, Lerman M, Sanders HJ: Ocular injuries in orbital fractures. A review of 119 cases. Plast Reconstruct Surg 1975, 56: 410-18.

5. Steidler NE, Cook RM, Reade PC: Residual complications in patients with major middle third facial fractures. Int $J$ Oral Surg 1980, 9: 259-66.

6. Luce EA, Tubb TD, Moore AM. Review of 1,000 major facial fractures and associated injuries. Plast Reconstruct Surg 1979, 63: 26-30.

7. Al-Qurainy IA, Stassen LFA, Dutton GN et al:: The characteristics of midfacial fractures and the association with ocular injury. Br J Oral Maxillofac Surg 1991 (in press).

8. Al-Qurainy IA, Stassen LFA, Dutton GN et al.: Symptomatic diplopia following midfacial fractures. $\mathrm{Br} J$ Oral Maxillofac Surg 1991 (in press).

9. Al-Qurainy IA, Titterington DM, Dutton GN et al. Midfacial fractures and the eye. The development of a system for detecting patients at risk of eye injury. Br J Oral Maxillofac Surg 1991 (in press).

10. Al-Qurainy IA, Dutton GN, Ilankovan V et al:: Mid-facial fractures and the eye. The development of a system for detecting patients at risk of eye injury. A prospective evaluation. Br J Oral Maxillofac Surg 1991 (in press). 